\title{
De la loi bioclimatique à la conception bioclimatique en architecture
}

\author{
Clément Gaillard \\ Institut de géographie, Université Paris 1, Paris \\ clement.gaillard@univ-paris1.fr
}

\section{Résumé}

Cet article souhaite étudier la circulation du terme « bioclimatique » à travers les différentes sciences où il a été employé au cours du $X X^{\mathrm{e}}$ siècle et les projets scientifiques auxquels il a été associé. Si aujourd'hui ce terme renvoie généralement à l'architecture, et à ce qu'on nomme la conception bioclimatique, il n'est pas issu de l'architecture puisqu'il provient des recherches d'un entomologiste américain du début du $\mathrm{XX}^{\mathrm{e}}$ siècle nommé Andrew D. Hopkins. D'abord utilisé par Hopkins, puis dans de nombreuses autres sciences par la suite, il a été popularisé avec le développement de la conception bioclimatique en architecture dès 1963. Cet article propose d'étudier comment les significations successivement associées à ce concept se sont manifestées dans les préoccupations propres à l'architecture.

$\mathrm{L}$ 'architecture traditionnelle et vernaculaire a souvent été considérée comme une expression du climat d'un lieu. Certains géographes ont ainsi étudié l'habitat populaire en proposant des corrélations entre les formes des constructions traditionnelles, la morphologie des toitures par exemple, et le type de climat où elles se situent (Dollfus, 1954). Alors que de nombreuses typologies de l'architecture vernaculaire peuvent être considérées comme adaptées au climat où sont situées ces constructions (Gaillard, 2020), la standardisation des techniques de construction au cours du $\mathrm{XX}^{\mathrm{e}}$ siècle a été vivement critiquée dans la mesure où elle aurait produit une architecture moderne inadaptée aux climats locaux, voire en contradiction flagrante avec certains facteurs climatiques (Alexandroff et Alexandroff, 1982).

Cependant, il s'est développé dans la seconde moitié du $\mathrm{XX}^{\mathrm{e}}$ siècle une méthode de conception en architecture consciente des dérives de l'architecture moderne, et soucieuse de se réapproprier certaines typologies de l'architecture traditionnelle par une considération explicite du climat et de ses propriétés : il s'agit de la conception bioclimatique. Bien qu'il existe des exemples de prise en compte du climat en architecture avant cette date, l'approche bioclimatique a été initialement développée dans les années 1950 aux États-Unis (Olgyay, 1963). Elle est présentée comme une méthode de conception et d'étude de l'architecture qui prend en compte les données climatiques locales afin de concevoir des bâtiments qui tirent profit des éléments climatiques favorables, et soient protégés de ses effets défavorables en fonction des saisons. Pour ce faire, cette méthode s'appuie sur l'étude qualitative du microclimat d'un site, ainsi que sur l'interprétation des données météorologiques de la station la plus représentative du climat local. La conception bioclimatique s'est développée durant les années 1970 et a connu un regain d'intérêt récent sous l'impulsion des différentes réglementations thermiques et énergétiques, dans la mesure où elle permet certaines économies de chauffage ou de climatisation (Cordier, 2013).

Si cette méthode est désormais appliquée par quelques ingénieurs, bureaux d'études et architectes, l'origine même du terme « bioclimatique » reste obscure et entretient de nombreuses confusions. Ce concept, aujourd'hui associé à l'architecture, n'est pas issu d'un architecte, mais a circulé dans de nombreuses sciences comme l'entomologie et la botanique, mais aussi la physiologie et la géographie humaine au cours du $X^{\mathrm{e}}$ siècle. Ce terme renvoie aux travaux d'un entomologiste, Andrew Delmar Hopkins, à l'origine de la loi bioclimatique formulée en 1918. Depuis Hopkins, on retrouve des traces de l'usage du terme « bioclimatique » dans d'autres travaux scientifiques correspondant à d'autres sciences, toujours liées à la climatologie ou à la météorologie. La circulation de ce concept jusqu'à son emploi en architecture nous paraît intéressante à retracer, afin de montrer qu'il a agrégé des problématiques scientifiques complexes relatives au climat et à ses effets. 


\section{Abstract}

\section{From "bioclimatic law" to "bioclimatic approach" in architecture}

This article aims to study the circulation of the term "bioclimatic" through the different sciences in which it has been used during the 20 th century and the scientific projects with which it has been associated. If today this term generally refers to architecture, and what is called bioclimatic design, it does not come from architecture since it comes from the research of an American entomologist at the beginning of the $20^{\text {th }}$ century named Andrew D. Hopkins. First used by Hopkins, then in many other sciences thereafter, it was popularized with the development of bioclimatic approach in architecture in 1963. This article proposes to study how the meanings successively associated with this concept were manifested. in the concerns specific to architecture.
Notre hypothèse est que les différentes significations associées au terme « bioclimatique » à travers ses emplois successifs se retrouvent indirectement dans l'approche bioclimatique en architecture, définie comme une méthode de conception, mais également de compréhension de l'architecture et des modes de vie à partir du climat. En examinant les différents emplois de ce terme, nous souhaitons par ailleurs mettre en avant la complexité de l'étude des effets du climat sur les êtres vivants, ainsi que la difficulté pour prendre en compte ces effets sans verser dans un déterminisme facile. Suivant un exposé chronologique, reposant sur l'étude de documents originaux, cet article vise à détailler les significations successives qui ont été associées au « bioclimatisme».

\section{La loi bioclimatique d'Andrew Delmar Hopkins}

Le terme " bioclimatique " semble synthétiser deux sciences préexistantes : la biologie et la climatologie. Or il n'appartient primitivement à aucune de ces deux disciplines. Il apparaît pour la première fois en langue anglaise en 1918 dans un long article de l'entomologiste américain Andrew Delmar Hopkins consacré à l'étude des phénomènes périodiques en agriculture (Hopkins, 1918). Andrew Hopkins (1857-1948) est d'abord un fermier passionné d'entomologie et plus particulièrement d'entomologie forestière. Ses premières études scientifiques concernent les insectes parasites responsables d'importants dégâts dans les forêts de pins, ainsi que les parasites du blé. C'est à partir de travaux sur les cycles de plantation du blé qu'il constate les importantes variations dans la croissance des semis en fonction de la latitude et de l'altitude (Hopkins, 1920). À la suite de ces observations, il émet l'hypothèse d'une influence dominante des facteurs et éléments climatiques sur la croissance des végétaux, notamment du rayonnement solaire, ainsi que l'influence des éléments géographiques comme la topographie, les lacs ou les cours d'eau sur la variation des phénomènes périodiques des végétaux (Hopkins, 1918). Ce sont ces observations et ces hypothèses qui l'amènent à énoncer sa « loi bioclimatique » en 1918, qu'il développe par la suite dans de nombreux autres articles.
Pour sa première occurrence, le terme « bioclimatique » n'apparaît pas isolément. L'article de 1918 présente en effet la loi bioclimatique (bioclimatic law) et c'est d'abord en tant que loi, au sens des lois déterministes de la physique classique, que ce terme est présenté. La loi bioclimatique désigne alors une loi générale de l'agronomie et de l'entomologie, permettant d'adapter les pratiques agricoles en fonction du climat local et du microclimat d'un lieu. Dans un article de 1920 qui est consacré à cette loi de la nature, Hopkins présente la loi bioclimatique en ces termes : « Les variations dans la date d'un événement périodique à partir d'une norme donnée ou d'une constante sont une mesure, en termes de temps, de l'intensité des influences dominantes et des forces en lien avec (a) la position géographique, (b) la saison, (c) la tendance inhérente des espèces sous les mêmes influences externes à varier entre des réponses individuelles précoces ou tardives, et (d) aux réponses précoces ou tardives des individus d'une même variété sous des influences locales variables. [...] Par conséquent, toutes choses étant égales par ailleurs, la variation entre deux au plusieurs positions géographiques est dans la même proportion que la distance qui les sépare, ainsi la durée de 4 jours correspond à 1 degré de latitude, 400 pieds d'altitude ou 5 degrés en longitude » (Hopkins, 1920).

En admettant qu'à une position dans l'espace en longitude, latitude ou altitude correspond un jeu de caractéristiques climatiques déterminées, il est alors possible de déduire d'une position géographique un retard ou une avance dans le temps d'un phénomène biologique périodique, toutes choses étant égales par ailleurs. La loi bioclimatique permet de calculer ces variations dans les phénomènes périodiques d'une ou plusieurs espèces végétales, en fonction de sa situation géographique (Hopkins, 1920). Pour ce faire, cette loi s'appuie sur d'abondantes observations effectuées en phénologie, qui est la science des phénomènes périodiques chez les êtres vivants.

Une des caractéristiques les plus intéressantes de la loi bioclimatique proposée par Hopkins concerne ce que nous pouvons appeler la réversibilité totale entre les conditions climatiques et les individus biologiques observés. En effet, si des facteurs climatiques déterminants influencent la croissance de certaines espèces, alors réciproquement l'observation de la croissance 
de certaines espèces permet de déterminer des influences climatiques. Hopkins écrit ainsi : «Le principe de base dans l'opération de cette loi se trouve dans le caractère des réponses des organismes aux éléments et facteurs complexes de son environnement local et immédiat. En d'autres termes, l'organisme est utilisé comme instrument par lequel le climat d'un lieu et les autres influences sur ses activités sont déterminés » (Hopkins, 1920).

L'idée qu'un organisme vivant puisse être pris comme un instrument de mesure des influences climatiques est originale. Cette réversibilité totale est essentiellement déterministe : des formes végétales conditionnées par des facteurs climatiques locaux, on peut déduire de l'observation de végétaux à un endroit donné l'ensemble des conditions climatiques signifiantes pour cette localité. Nous retrouverons cette réversibilité dans les travaux des architectes bioclimatiques et particulièrement dans ceux d'Alain Guyot en France sur l'influence du vent sur la forme de la végétation (Izard et Guyot, 1979).

La loi bioclimatique ne semble pas avoir eu d'applications pratiques sur la production agricole comme l'avait envisagé Hopkins, bien que ses recherches se soient orientées dans ce sens, en proposant notamment une classification des zones bioclimatiques (Hopkins, 1921). En 1925, paraît en français dans la revue La Météorologie, un court article de Hopkins intitulé « Notes sur la Loi Bioclimatique » en réponse à un appel pour la coopération internationale des recherches en phénologie. Il mentionne le projet d'une bioclimatologie, fondée sur la loi bioclimatique, dont l'intérêt est d'exprimer la « répercussion de la causalité représentée par le soleil, le mouvement de la Terre, la répartition inégale des terres et des mers, et de la physiographie variée des terres insulaires et continentales » (Hopkins, 1925). Initialement pensée à l'intersection entre la botanique, la phénologie, l'agriculture, la climatologie et la météorologie, la loi bioclimatique est encore généralisée en 1925 par Hopkins pour y inclure « l'étude de la géographie de la vie humaine et du climat » menée en géographie humaine. Malgré l'intérêt de ses recherches et la publication d'un ouvrage consacré aux « bioclimatiques » par Hopkins en 1938 (Hopkins, 1938), l'usage de la loi bioclimatique ne s'est que timidement développé par la suite.

\section{L'étude des micro- climats et la variation des phénomènes périodiques}

En dépit des faibles développements de la loi bioclimatique, l'intérêt pour la variation des phénomènes biologiques périodiques en fonction du climat, dont on peut trouver des traces dans les travaux plus anciens d'Alexander van Humboldt, s'est cependant poursuivi au-delà des travaux de Hopkins. Ses préoccupations se sont retrouvées dans celles relatives à l'étude des microclimats entreprise entre autres par le climatologue Rudolf Geiger en Allemagne et par le météorologue Helmut Landsberg aux États-Unis. Geiger est l'auteur d'un ouvrage consacré à l'étude des climats près du sol publié en 1927 et dont la traduction en anglais intitulée The Climate near the Ground publiée en 1950 est mentionnée comme le premier traité explicitement consacré à l'étude des microclimats. Sans mentionner les travaux de Hopkins, Geiger s'intéresse à des problèmes analogues, relatifs aux variations induites par le microclimat sur la croissance des végétaux (Geiger, 1950). À la différence de Hopkins qui avait imaginé modifier les pratiques agricoles grâce à la loi bioclimatique, le but de Geiger est d'encourager l'étude des microclimats afin de tirer parti des phénomènes microclimatiques pour protéger et aménager les cultures (Geiger, 1950).

À la même période, le météorologue américain Helmut Landsberg reprend les recherches de Geiger et développe l'étude des microclimats dans le champ de l'architecture et de l'urbanisme dès 1947. En 1949, il publie un article intitulé « Vivre confortablement dépend du microclimat » (Comfortable living depends on microclimate) dans un périodique d'architecture largement diffusé (Landsberg, 1950). Cet article propose aux particuliers de développer leur connaissance du microclimat où se situe leur maison afin d'en améliorer le confort et éventuellement de réduire les dépenses liées au chauffage ou à la climatisation de leur habitation. Il propose les conseils suivants afin d'étudier le microclimat : « Relevez les différentes dissemblances climatiques du quartier où vous habitez par rapport aux stations officielles, en toutes saisons. Observez les différences dans la vie des plantes, en particulier les dates de première floraison au printemps. Elles offrent de nombreux indices utiles sur le microclimat. Regardez les effets microclimatiques des arbres, des arbustes, des haies. Notez les différences de pentes des diverses expositions et les différences entre le sommet des collines et les vallées. Regardez les influences des surfaces d'eau. Notez les différences de température de diverses surfaces. Prenez la mesure des températures à l'intérieur et à l'extérieur dans divers contextes. Il est généralement facile de mettre à profit les connaissances ainsi acquises pour améliorer le microclimat de votre propre maison » (Landsberg, 1950).

Dans cet extrait, on remarque que Landsberg évoque l'importance de relever les dates de floraison afin d'en déduire des « indices utiles sur le microclimat ». Ce type de renseignement relève directement de la phénologie empirique, et la loi bioclimatique établie par Hopkins devait en théorie permettre de déduire l'avance ou le retard dans les cycles de floraison, à partir de la latitude, la longitude et l'altitude du lieu considéré. Sans mentionner les travaux de Hopkins, Landsberg propose d'utiliser la végétation comme un « instrument » susceptible de fournir des renseignements sur le microclimat, dans la continuité indirecte des recherches de Hopkins.

On retrouve dans ces recherches en microclimatologie d'autres préoccupations analogues à celles de Hopkins. En 1954, Landsberg publie un article intitulé " Bioclimatologie du logement » (Bioclimatology of housing) dans un volume consacré aux travaux en bioclimatologie menés par un groupe de recherche auquel participent des biologistes, des physiologistes et des géographes. Dans la préface au volume regroupant ces travaux, le géographe américain Jacques M. May, spécialisé en géographie médicale, présente la bioclimatologie comme « cette branche de l'écologie qui s'intéresse aux effets de l'environnement physique sur les organismes vivants » et se réfère à Hippocrate ainsi qu'à Pasteur (May, 1954). Cette approche des faits géographiques par l'écologie biologique est aussi partagée par le géographe Maximilien Sorre en France. Tout en faisant référence aux travaux de Geiger, May souligne la diversité des microclimats produisant un environnement physique différencié pour chaque être vivant : "Les climats varient non seulement entre les pôles et l'équateur, entre le niveau de la mer et les sommets des montagnes, mais entre un creux aussi grand que la paume de la main dans un champ 
et une dépression similaire à quelques mètres. Toutes ces variations se produisent selon des lois naturelles, dont certaines sont découvertes et apprises par l'homme, dont certaines restent mystérieuses et représentent le champ de recherche de demain » (May, 1954).

Ces propos sont analogues à ceux écrits par Hopkins plus de trente années auparavant, notamment le fait que May affirme que les variations observées en bioclimatologie obéissent à des lois naturelles qu'il est possible de découvrir, Hopkins estimant que la loi bioclimatique était une des lois de la nature (Hopkins, 1920).

Cependant, on remarque que le domaine d'étude de la bioclimatologie a gagné en extension par rapport aux travaux d'Hopkins. L'article de Landsberg consacré à la bioclimatologie du logement porte exclusivement sur l'étude de l'habitation, qui est un environnement physique artificiel produit par l'action de l'homme et partiellement soustrait à l'action du climat local. Alors qu'Hopkins pouvait émettre des recommandations relatives aux pratiques agricoles à partir de la loi bioclimatique, l'étude bioclimatique des logements proposée par Landsberg permet d'émettre des prescriptions relatives aux choix effectués par les architectes et les ingénieurs. Il estime que si la plupart des individus passent la majorité de leur temps de vie en intérieur, dans un climat modifié par l'action d'une structure construite qu'il nomme " cryptoclimat ", l'étude bioclimatique des habitations et des lieux clos est primordiale (Landsberg, 1954). Son article passe en revue l'action de différents facteurs climatiques (ensoleillement, précipitations, vents...) sur les constructions et aboutit à des considérations relatives « au confort à l'intérieur des habitations », comme les impératifs de renouvellement d'air. Dans ses analyses, Landsberg reprend la notion de zone de confort développée par les ingénieurs à partir des mesures de la température, de l'humidité relative et de la vitesse de l'air (Landsberg, 1954). C'est à partir de ces considérations bioclimatiques sur la zone de confort que va se développer l'approche bioclimatique en architecture.

\section{Physiologie et développement de l'approche bioclimatique}

Les travaux de Landsberg en bioclimatologie et sur l'étude des microclimats ont considérablement influencé certains architectes américains, notamment les frères Victor et Aladár Olgyay qui ont collaboré avec Landsberg dès le début des années 1950. Soucieux de concevoir des bâtiments adaptés au climat local, dans la perspective de réduire les coûts de chauffage et de climatisation, ces architectes ont entamé dès les années 1950 la mise au point de différentes méthodes de conception en architecture, aboutissant à ce que Victor Olgyay nomme l'approche bioclimatique (Olgyay, 1963). Olgyay montre l'intérêt d'utiliser ce qu'il nomme les « diagrammes bioclimatiques ", qui sont des outils développés par les physiologistes et qui ont été adaptés aux usages de l'architecte (figure 1).

À partir de données météorologiques horaires pour chaque mois, ces diagrammes permettent de repérer et de caractériser visuellement un climat par rapport à la zone de confort établie conventionnellement, l'axe des abscisses représentant l'humidité relative, et celui des ordonnées la température sèche. Les données météorologiques de la région de Minneapolis, par exemple, montrent que les températures y sont trop basses la majorité de l'année par rapport à la zone de confort (figure 2). Le diagramme bioclimatique permet de lire un certain nombre de corrections susceptibles de ramener des valeurs en dehors de la zone de confort à l'intérieur de celle-ci : des températures trop froides peuvent être ressenties comme confortables par rayonnement d'une certaine quantité d'énergie thermique (partie inférieure du diagramme), tandis que l'inconfort produit par des températures trop

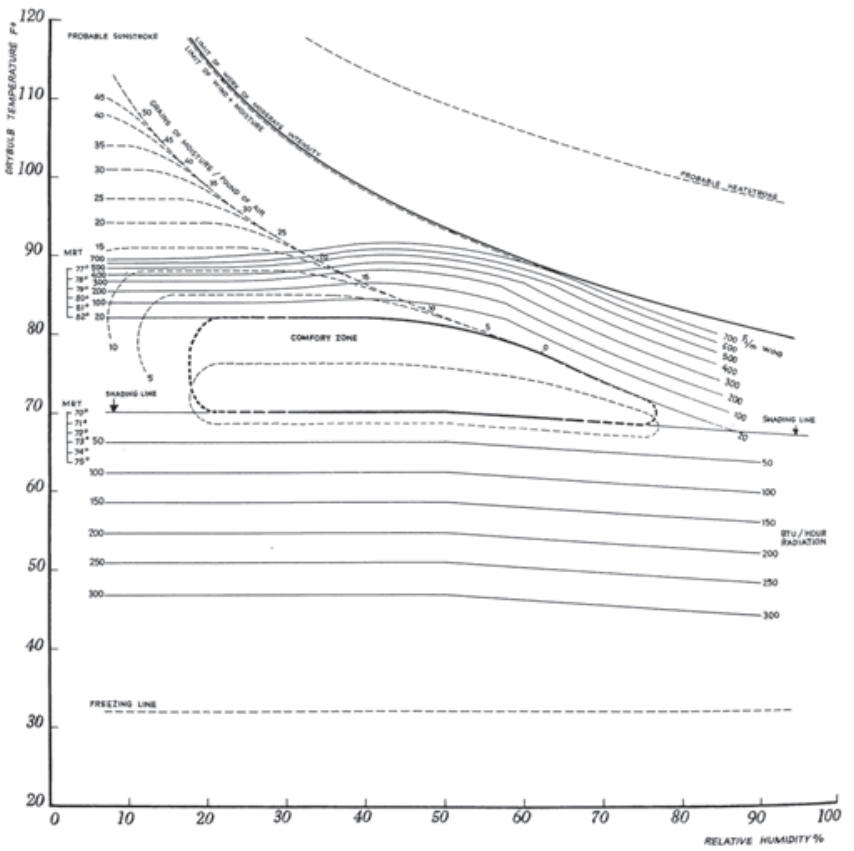

Figure 1. Le diagramme bioclimatique d'Olgyay (1963), la zone confort est entourée d'un ensemble de graduations qui représentent les moyens de produire une sensation de confort (vitesse de l'air, rayonnement...).

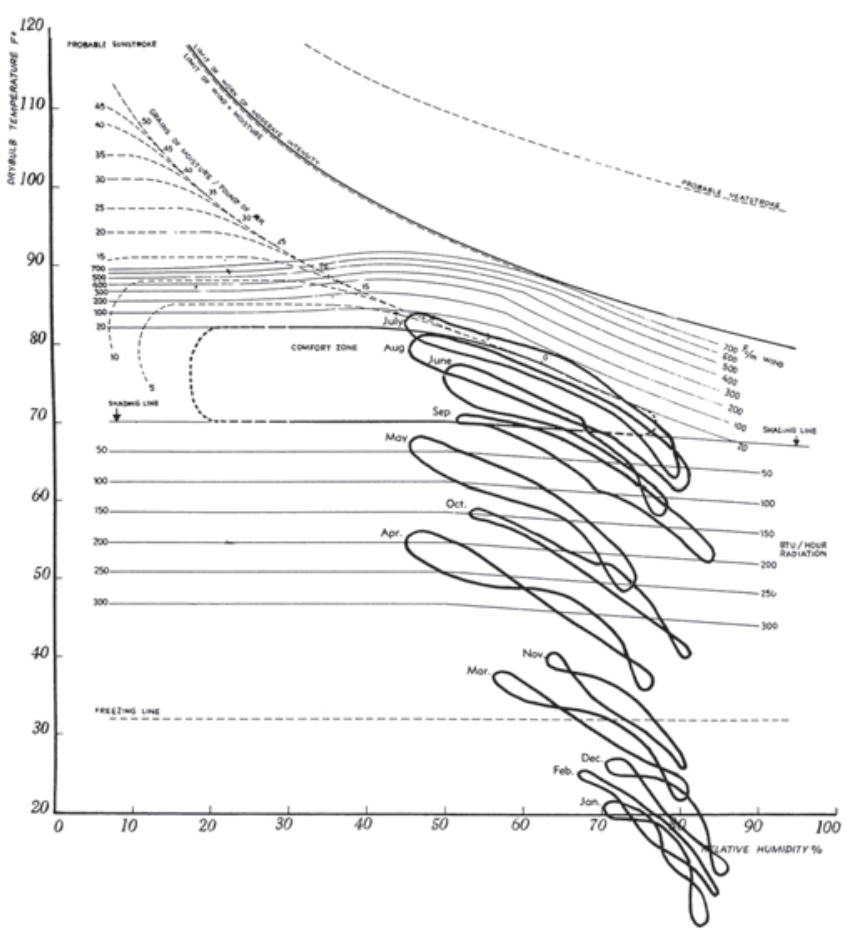

Figure 2. Le diagramme bioclimatique d'Olgyay (1963) avec le repérage des données mensuelles pour la zone de Minneapolis. 
chaudes et une humidité relative trop importante peut être tempéré par une vitesse de l'air élevée (partie supérieure du diagramme). L'architecte peut ainsi visualiser les périodes durant lesquelles le climat impose certaines dispositions architecturales, comme favoriser la pénétration du rayonnement solaire en hiver ou améliorer la ventilation naturelle en été.

Le diagramme bioclimatique développé par Victor et Aladár Olgyay est un outil de diagnostic d'un climat, permettant d'anticiper l'inconfort possible dans un climat donné et d'en déduire certaines solutions architecturales. C'est cet outil qui donne son nom à l'approche bioclimatique imaginée par Olgyay en 1963, celle-ci étant à l'origine de la conception bioclimatique en architecture qui s'est développée dès le début des années 1970. À partir de cette période, ces diagrammes seront très largement reproduits, modifiés et commentés par les architectes (Izard et Guyot, 1979). De par le caractère analytique de cette démarche, qui fait appel à de nombreux outils graphiques permettant de déduire des solutions architecturales d'un climat local, le " bioclimatisme » sera même considéré comme une science par certains architectes (Izard et Guyot, 1979). Bien que cet outil ne fût que rarement utilisé en pratique, la conception bioclimatique en architecture suit une démarche analogue à celle proposée par Olgyay : elle déduit de données météorologiques et d'observations climatiques des solutions architecturales susceptibles d'assurer le confort, par un recours minimum à la climatisation et au chauffage, en maximisant les apports climatiques favorables. Les maisons bioclimatiques de cette période peuvent être caractérisées par l'utilisation de larges serres orientées au sud pour favoriser les apports d'hiver (figure 3), ou de systèmes conçus pour capter et accumuler l'énergie solaire pour le chauffage (figure 4). Elles sont aussi caractérisées par l'utilisation de protections contre le rayonnement solaire ou les vents dominants sur les façades les plus défavorablement orientées (figure 5).

À l'origine, la conception bioclimatique est également une approche de l'architecture et s'inscrit plus généralement dans une compréhension des phénomènes biologiques considérés du point de vue des conditions climatiques qui permettent leur développement. En plus de mentionner les relations entre végétation et microclimat, Victor Olgyay prend pour exemple la forme

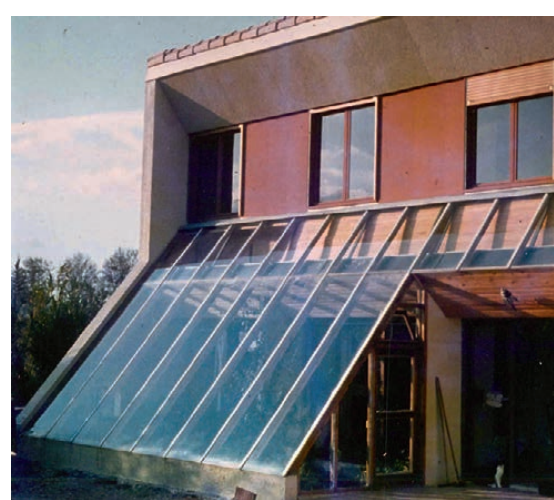

Figure 3. Maison à Ginestas (Aude), en 1980. Architecte : Michel Gerber. Photographie : Lola Vico-Gerber.

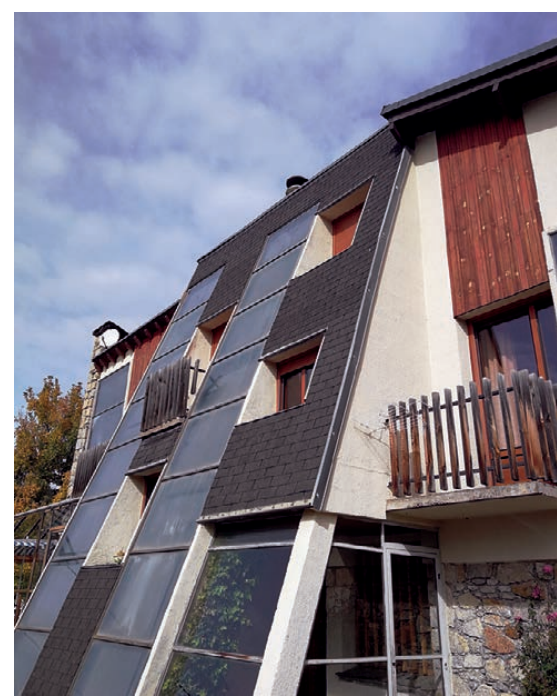

Figure 4. Maison équipée de murs Trombe à Odeillo-Font-Romeu (Pyrénées-Orientales), en 1980. Architecte et ingénieur : Jean-François Tricaud.

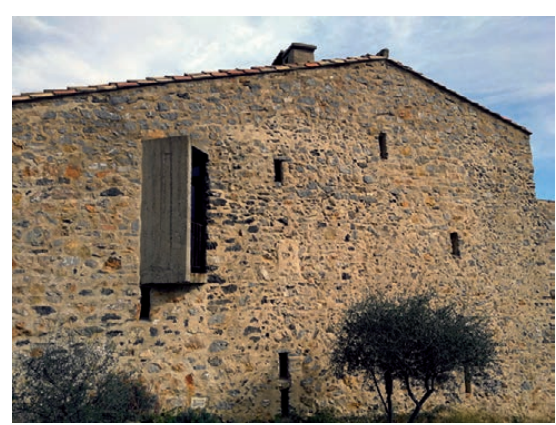

Figure 5. Façade ouest exposée au vent d'une maison rénovée à Treilles (Aude), en 1979. Architecte : Michel Gerber.

des feuilles de certains types de végétaux dans différents types de climats, qui minimisent leurs surfaces pour amoindrir les effets du rayonnement solaire (Olgyay, 1963). D'autres architectes ayant étudié l'orientation des constructions émettront l'idée que la disposition des parcelles agricoles est source d'enseignement pour l'architecte, celles-ci bénéficiant le plus souvent d'un ensoleillement favorable en hiver (Grillo, 1975). L'approche bioclimatique en architecture est intégrée à une compréhension plus générale des caractéristiques bioclimatiques, dans la lignée indirecte de la bioclimatologie imaginée par certains géographes.

Que reste-t-il alors du projet de Hopkins? Il est frappant de voir une grande proximité entre ses recherches et celles de certains architectes ayant travaillé sur la conception bioclimatique en France. Pour Hopkins, les végétaux peuvent être utilisés comme des « instruments » renseignant sur des influences climatiques dominantes (Hopkins, 1920). Cette idée apparaît dans les recherches sur les effets du vent dans la conception des bâtiments menées par Alain Guyot, dans le cadre du groupe ABC (Ambiances bioclimatique en architecture) à l'école d'architecture de Marseille-Luminy. Dans le chapitre consacré au vent du manuel Archi bio co-écrit avec JeanLouis Izard, Alain Guyot affirme que les végétaux constituent un indicateur permanent pour comprendre les effets du vent dans une localité donné. Il écrit : " [Les indicateurs permanents] donnent une image définitive (ou presque) d'un état engendré par le vent. Parmi les différents cas énumérés plus loin, l'élément végétal possède un pouvoir d'information des plus signifiants, car il s'agit d'un élément vivant qui subit les actions du vent. Celles-ci influent sur la croissance qui témoigne alors des adaptations aux conditions locales. Cette classe d'indicateurs permet de dégager les effets les plus contraignants sous un aspect fréquentiel » (Izard et Guyot, 1979).

Comme l'affirmait Hopkins, l'observation de la végétation permet de déduire certains phénomènes microclimatiques. L'architecte doit donc être en mesure d'interpréter la végétation afin de mieux comprendre le microclimat où il souhaite construire. Guyot évoque également le maillage agricole de la plaine de Crau qui permet une bonne protection des cultures contre les effets du Mistral (Izard et Guyot, 1979).

\section{Conclusion : la généralité de l'approche bioclimatique}

Le parallèle entre la loi bioclimatique d'Hopkins et le développement de l'approche bioclimatique par la suite permet de mettre en avant une certaine continuité dans l'étude des 
productions naturelles ou artificielles en relation avec le climat local où elles se situent. Contrairement à ce que l'architecte Jean-Louis Izard affirme, Victor Olgyay n'est pas le créateur du terme " bioclimatique » (Izard et Guyot, 1979), il a repris l'étude bioclimatique du confort développée par des physiologistes, elle-même issue des recherches en microclimatologie et en agriculture initiées par Hopkins.

En devenant une méthode de conception en architecture, l'approche bioclimatique inaugurée par Olgyay a indirectement hérité des problématiques soulevées dans l'ensemble des travaux de recherche relatifs aux effets du climat sur les êtres vivants et leurs habitats, des termitières, qui fournissent des leçons d'orientation au climatologue (Geiger, 1950) comme à l'architecte (Olgyay, 1963), jusqu'aux microclimats complexes créés dans les agglomérations urbaines. Malheureusement, la conception bioclimatique a pu être réduite au cours de son développement à une simple application de principes censés réaliser des économies d'énergie, notamment de chauffage, en négligeant que le bioclimatisme auquel elle se réfère agrège des problématiques qui ont été partagées par de nombreuses sciences, comme la géographie humaine, la climatologie ou l'agriculture au cours du XX $X^{\mathrm{e}}$ siècle. À l'inverse de cette perspective, qui présuppose que les effets du climat sur les êtres vivants et les modes de vie sont définitivement compris, nous espérons avoir pu montrer que le développement de l'approche bioclimatique en architecture s'inscrit dans un projet scientifique plus général qui vise à clarifier l'influence du climat sur les formes de vie et qui, à ce titre, est d'une grande actualité pour nous aujourd'hui.

\section{Bibliographie}

Alexandroff G., Alexandroff J.-M., 1982. Architectures et climats. Soleil et énergies naturelles dans I'habitat. Berger-Levrault, Paris, 378 p.

Cordier J.-P. (dir.), 2013. Conception architecturale et RT 2012. Influence des facteurs énergétiques sur la forme des bâtiments. Le Moniteur, Paris, 300 p.

Dollfus J., 1954. Les Aspects de l'architecture populaire dans le monde. Albert Morancé, Paris, 134 p.

Gaillard C., 2020. L'approche énergétique de l'architecture vernaculaire : genèse et développement. Socio-Anthropologie, 42, 67-80. doi: 10.4000/socio-anthropologie.7372

Grillo P.J., 1975. Form, Function \& Design. Dover Publication Inc., New York, 238 p.

Geiger R., 1950. The Climate near the Ground. Harvard University Press, Cambridge, trad. de l'allemand par Milroy N. Stewart et al., 482 p.

Hopkins A.D., 1918. Periodical Events and Natural Law as Guides to Agricultural Research and Practice. Supplement to Monthly Weather Review, No. 9, U.S. Department of Agriculture, Washington, $42 \mathrm{p}$.

Hopkins A.D., 1920. The bioclimatic law. Journal of the Washington Academy of Sciences, Vol. X, Washington Academy of Science, Washington, 34-40.

Hopkins A.D., 1921. Bioclimatic zones of the continents; with proposed designations and classification. Journal of the Washington Academy of Sciences, Vol. XI, Washington Academy of Science, Washington, 227-229.

Hopkins A.D., 1925. Notes sur la Loi Bioclimatique. La Météorologie. Revue de météorologie et de physique du globe, Tome XVI, Etienne Chiron, Paris, $70-71$.

Hopkins A.D., 1938. Bioclimatics: A Science of Life and Climate Relations. United State of Agriculture, United State Government Printing Office, Washington, 188 p.

Izard J.-L., Guyot A., 1979. Archi bio. Parenthèses, Marseille, 131 p.

Landsberd H.E., 1950. Comfortable living depends on microclimate, Weatherwise. 3, 7-10.

Landsberg H.E., 1954. Bioclimatology of housing. Wendell E. (éd.), Meteorological Monograph. Recent Study in Bioclimatology: a group, Vol. 2, No. 8, American Meteorological Society, Boston, 81-98

May J.M., 1954. Preface, Wendell E. (éd.), Meteorological Monograph. Recent Study in Bioclimatology: a group, Vol. 2, No. 8, American Meteorological Society, Boston, III-IV. Olgyay V., 1963. Design with Climate. Bioclimatic Approach to Architectural Regionalism. Princeton University Press, Princeton, 224 p. 